\title{
Checklist of plants of Rashad and Alabassia localities (eastern Nuba Mountains), South Kordofan Sudan
}

\author{
Ismail Mirghani Ismail $^{1 *}$ and Alawia Abdalla Elawad ${ }^{2}$ \\ 1 Agricultural Research Corporation, Forestry Research Centre, P.O. Box 13391, Khartoum, Sudan \\ 2 Department of environmental sceinces, Faculty of Sciences and Technology, AlNeelain University, Khartoum, P.O. Box 12702, Sudan \\ * Corresponding author. E-mail address: ismail.mirghani@yahoo.com
}

\begin{abstract}
This study identifies and documents species of the native flora the low rainfall woodland savanna of Rashad and Alabassia localities (eastern Nuba Mountains), Sudan. This study identified 260 species belonging to 176 genera and 59 families, including five new records to the flora of the Sudan. The flora is comprised of a variety of different life-forms. Most are phanerophytes $40 \%$, therophytes $35 \%$, and chamaephytes $21 \%$; the remaining are geophytes, hydrophytes, hemicryptophytes and epiphytes. The majority of the plant species have important economic uses.
\end{abstract}

Key words: flora, vegetation of Sudan, biodiversity, lifeforms, economic uses

\section{INTRODUCTION}

A thorough knowledge of the flora of a certain area is an essential prerequisite to sound land-use policy. The present study fills a gap in our knowledge of the flora of Rashad and Alabassia localities, eastern Nuba Mountains, Sudan. Furthermore, this contribution provides a reliable scientific basis for the assessment of the production potential and management of plant resources in the study area.

The flora of the Nuba Mountains is rarely covered in literature, either on its own or as part of the larger Sudan flora. The earliest collections from the area was reported by Massey (1926) who recorded three grasses. Shortly thereafter, Broun and Massey (1929) included 128 plant species from the area. In works of Andrews (1950, 1952, 1956) only 24 plant species from the area were recorded. Elamin's (1990) publication on the trees and shrubs of the Sudan included 66 woody plant species from the Nuba Mountains.

The flora of certain locations, including some special habitats, in the Nuba Mountains has been studied. El Ghazali (1985) recorded 178 medicinal plants from the eastern Nuba Mountains. Ismail (2007) studied the woody vegetation of Rashad district and recorded 87 indigenous species and 15 exotic species.

The region in the eastern Nuba Mountains ,selected for the present study, exhibits wide topographical variation which is reflected in the diversity of vegetation. This study aims to identify and document the flora of the eastern Nuba Mountains and consequently to contribute in updating the flora of Sudan. Vouchered specimens also contribute the reference collection in the Forestry Research Centre herbarium at Soba, Sudan.

\section{MATERIALS AND METHODS Study area}

The study area is located in the northern part of the eastern Nuba Mountains of South Kordofan state, Sudan, and includes two localities (Rashad and Alabassia), extending from $11^{\circ} 33^{\prime}$ to $12^{\circ} 33^{\prime} \mathrm{N}$ and from $031^{\circ} \mathrm{O} 8^{\prime}$ to $031^{\circ} 18^{\prime} \mathrm{E}$. (Figure 1). Most of study has scattered isolated hills and it is dissected by many seasonal watercourses. The Rashad and Alabassia localities occupy an area of 7872 $\mathrm{km}^{2}$ (Adam et al. 2012). The study area classified as low rainfall woodland savanna (Harrison and Jackson 1958).

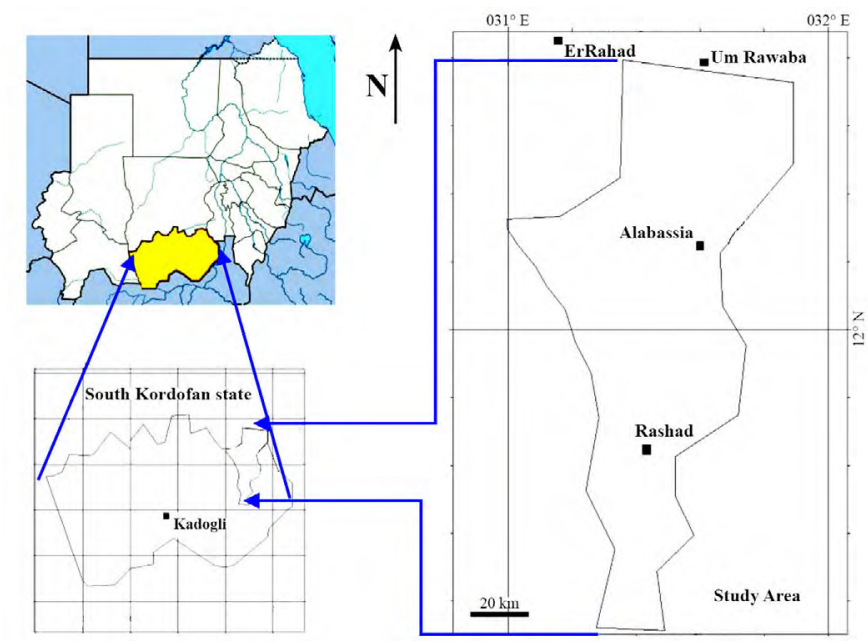

Figure 1. Study area: Rashad and Alabassia localities, South Kordofan state, Sudan. 


\section{Specimen collection and identification}

Plant specimens were collected during 2010-2011. The collection procedure followed methods described by Lawrence (1969) and Forman and Bridson (1991). Specimens were identified using Broun and Massey (1929), Andrews (1950, 1952 and 1956), and Elamin (1983, 1990). Identified specimens were confirmed with authenticated herbarium specimens in the Forestry Research Centre Herbarium at Soba.

Recent literature was consulted for current plant names: Friis and Vollesen (1998, 2005) and The Plant List (2013). The list of families covered in this study was arranged according to the Linear Angiosperm Phylogeny Group (LAPG) III (Haston et al. 2009), while subfamilies, genera, and species are arranged alphabetically within the families. Vernacular names and economic uses given were compiled from local people and available literature. The life-forms of plants were based on Raunkiaer (1934).

\section{RESULTS}

A detailed botanical account on the flora is given in the form of a checklist. Altogether 260 plant species, beloning to 59 families and 176 genera (Table 1) were identified. This number exceeds the number of species recorded previously from the Nuba Mountains.

The flora is grouped into 11 clades. Most of the species

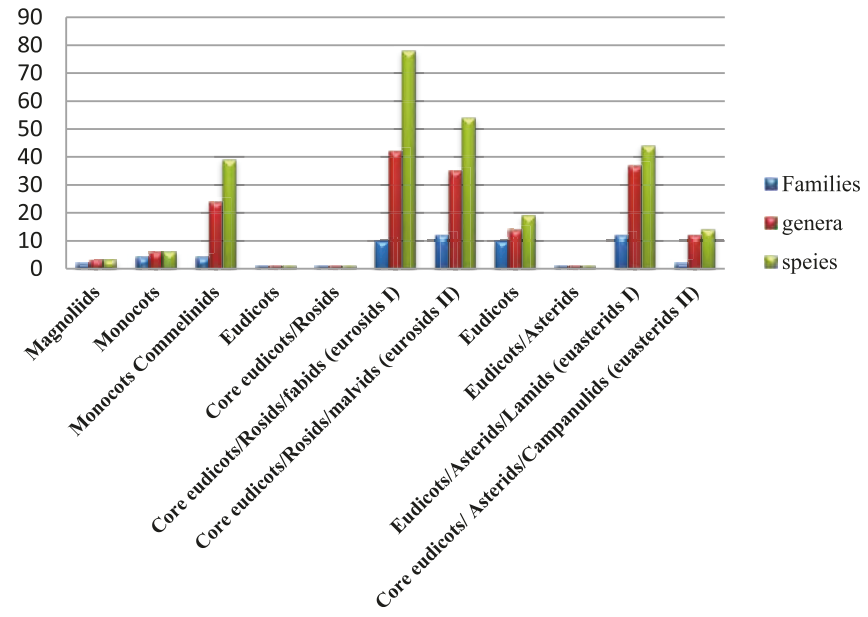

Figure 2. The plant species, genera and families included by clades.

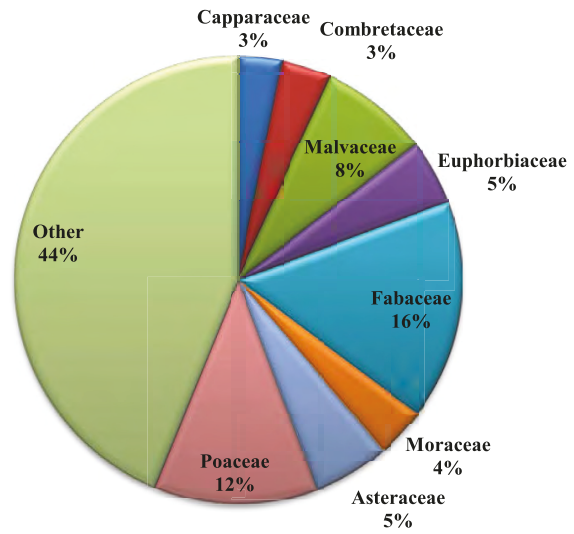

Figure 3. Distribution of plant species by family. belong to the core eudicots/rosids/fabids (eurosids I) (78 species, 42 genera and 10 families); core eudicots/ rosids/malvids (eurosids II) (54 species, 35 genera and 12 families); eudicots/asterids/lamids (euasterids I) (44 species, 37 genera and 12 families); monocots commelinids (39 species, 24 genera and four families) and each of the other clades include less than 20 species (Figure 2). The families Fabaceae (Leguminosae), Poaceae and Malvaceae are well represented in the flora of South Kordofan. Asteraceae and Euphorbiaceae are moderately present, while other families had between one and nine species (Figure 3).

In this study, five species were added to the flora of Sudan: Ludwigia adscendens (Onagraceae), Commicarpus chinensis (Nyctaginaceae), Crotalaria lanceolata (Fabaceae), Goniocaulon indicum (Asteraceae) and Tetrapogon tenuellus (Poaceae).

\section{DISCUSSION}

Most of the plant species of the study area have important economic uses. The study area is considered as important sources for animal wellbeing; $26 \%$ of the species are fodder plants. However, an important alternative to fodder is woody vegetation, which is browsed by animals during the dry season. Both herbaceous and woody plants (39\%) are used for medicinal purposes. Large trees and shrubs ( $7 \%$ of total flora) are utilized for timber that is used for making furniture, tool handles, and as building material (roofing, fencing, etc.). Timber is used for building material and other domestic uses such as roofing, fencing and as tool handles. Species used for fuel wood (8\%) are important source sof energy. Plants (15\%) also have edible fruits that are important during periods of famine. Some species (2\%) produce gums: gum arabic from Acacia senegal and frankincense gum from Boswellia papyrifera and Commiphora africana Figure (5).

\section{Endangered species}

This survey found that a number of economically important woody plants may be endangered. This includes Borassus aethiopum, a tree that is extensively cut for roof poles when mature; its germinating seeds are also dug for food ("Halook").. It is now only in protected

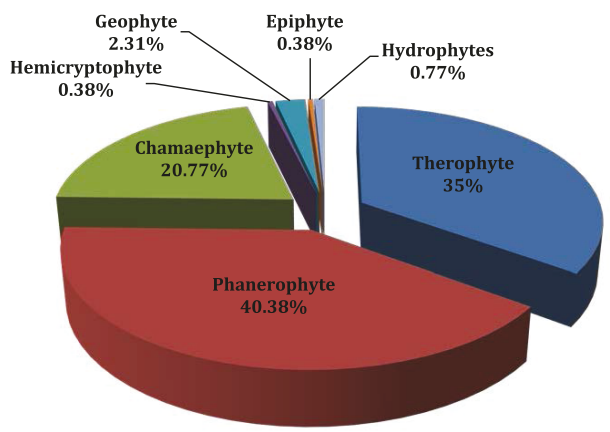

Figure 4. Life-form categories. 


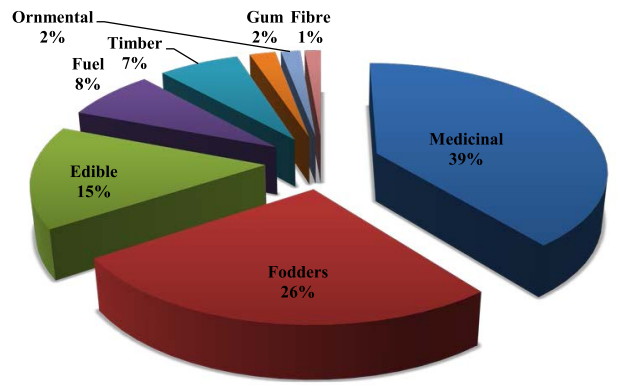

Figure 5. Economic uses of plant species.

areas such as gardens and around houses. Hyphaene thebaica was found only around houses as well. Cordia africana was observed only in gardens and aorund houses but were growing naturally. No regeneration of Boswellia papyrifera was recorded at the sites studied.

\section{ACKNOWLEDGEMENTS}

We are grateful to Prof. Ekhlas A. Bari (Environmental Studies Center, Qatar University) for revision and valuable comments and suggestions on the manuscript.

\section{LITERATURE CITED}

Adam, Y.O., M.A. Eisa, and D.M. Ahmed. 2012. Role of Adansonia digitata L. fruits in development and forest conservation in Rashad locality, Nuba Mountains, Sudan. University of Khartoum Journal Agricultural Sciences 20(3): 314-328.

Andrews, F.W. 1950. The flowering plants of the Angl-Egyptian Sudan, Vol. 1. Arbroath, UK: Buncle \& Co. 237 pp.

Andrews, F.W. 1952. The flowering plants of the Angl-Egyptian Sudan, Vol. 2. Arbroath, UK: Buncle \& Co. 482 pp.

Andrews, F.W. 1956. The flowering plants of the Sudan, Vol. 3. Arbroath, UK: Buncle \& Co. 579 pp.

APG III. 2009. An update of the Angiosperm Phylogeny Group classification for the orders and families of flowering plants: APG III. Botanical Journal of the Linnaean Society 161: 105-121. doi: 10.1111/j.1095-8339.2009.00996.x

Broun, A.F. and R.E. Massey. 1929. Flora of the Sudan. London: The Controller, Sudan Government Office. 494 pp.

Elamin, H.M. 1983. Trees and shrubs of Sudan [Ph.D. thesis].
Khartoum: University of Khartoum. 1033 pp.

Elamin, H.M. 1990. Trees and shrubs of the Sudan. Exeter, UK: Ithaca Press. 484 pp.

Elghazali, G.E.B. 1985.The flora of the eastern Nuba Mountains with special reference to medicinal plants [M.Sc. thesis]. Khartoum: University of Khartoum. 275 pp.

Forman, L. and D. Bridson. 1991. The herbarium handbook. Kew: Royal Botanic Gardens. 214 pp.

Friis, I.B. and K.A.J. Vollesen.1998. Flora of the Sudan-Uganda border area east of the Nile.Catalogue of vascular plants, $1^{\text {st }}$ part. The Royal Danish Academy of Sciences and Letters, Copenhagen. Biologiske Skrifter 51(1): 1-398.

Friis, I.B. and K.A.J. Vollesen. 2005. Flora of the Sudan-Uganda border area, east of the Nile. II. Catalogue of vascular plants, $2^{\text {nd }}$ part, vegetation and phytogeography. The Royal Danish Academy of Sciences and Letters, Copenhagen. Biologiske Skrifter 51(2): 399-855.

Harrison, M.N. and J.K. Jackson.1958. Ecological classification of the vegetation of the Sudan. Agricultural Publication Committee, Khartoum. 44 pp.

Haston, E., J.E.Richardson, P.F.Stevens, M.W.Chase and D.J. Harris. 2009. The Linear Angiosperm Phylogeny Group (LAPG) III: a linear sequence of the families in APG III.Botanical Journal of the Linnaean Society 161: 128-131. doi: 10.1111/j.10958339.2009.01000.x

Lawrence, G.H.M. 1969. Taxonomy of vascular plants. Calcutta/ Bombay/New Delhi: IBH Publishing. 823 pp.

Massey, R.E. 1926. Sudan grasses. Botanical series, publication no.1. Khartoum: McCorouodale \& Co. 55 pp.

Raunkiaer, C. 1934. The life form of plants and statistical plant geography. Oxford: The Clarendon Press. 632 pp.

The Plant List. 2013. The plant list. Version 1.1. Accessed at http:// www.theplantlist.org, 1 January 2013.

Vogt, K. 1995. Common trees and shrubs of dryland Sudan. A field workers guide to the identification, propagation and uses, SOS. London: Sahel International. $167 \mathrm{pp}$.

Authors' contribution statement: IMI collected the data, IMI and AAE identified and classified collected plant species and wrote the text.

Received: 10 April 2015

Accepted: 4 June 2015

Academic editor: Nik Fadzly

Table 1. Checklist of Flora of Rashad and Alabassia localities, Nuba Mountains, Sudan. Life forms: Ph = phanerophyte, Th $=$ therophyte, $\mathrm{Ch}=\mathrm{chamae}-$ phyte, $\mathrm{G}=$ geophytes, $\mathrm{Hy}$ = hydrophyte, $\mathrm{He}=$ hemicryptophyte, $\mathrm{Ep}=$ epiphyte. Economic uses: $\mathrm{Fd}=$ fodder, $\mathrm{M}=$ medicinal, $\mathrm{T}=$ timber, Fu = Fuel wood, Ef = edible fruit, $\mathrm{G}=$ gum.

\begin{tabular}{|c|c|c|c|c|}
\hline Taxa & Species & Vernacular name & Life form & Uses \\
\hline \multicolumn{5}{|l|}{ Clade Magnoliids } \\
\hline \multicolumn{5}{|l|}{ Order Piperales } \\
\hline \multicolumn{5}{|l|}{ Family Aristolochiaceae } \\
\hline Subfamily Aristolochioideae & Aristolochia bracteolata Lam. & Um Jalajil, & $\mathrm{Ch}$ & M \\
\hline \multicolumn{5}{|l|}{ Order Magnoliales } \\
\hline \multirow[t]{2}{*}{ Family Annonaceae } & Annona senegalensis Per. & Harhar, Gishta & $\mathrm{Ph}$ & $\mathrm{T}, \mathrm{M}, \mathrm{E}$ \\
\hline & Xylopia acutiflora (Dunal) A. Rich. & Kurnagang & $\mathrm{Ph}$ & $\mathrm{T}, \mathrm{M}, \mathrm{E}$ \\
\hline
\end{tabular}

\section{Clade Monocots}

Order Alismatales

Family Araceae

Subfamily Aroideae

Amorphophallus abyssinicus (A. Rich.) N. E. Br.

Pistia stratiotes L.

Stylochaeton hypogaeus Lepr.

$\begin{array}{lll} & \text { G } & \text { E } \\ & \text { Hy } & M \\ \text { Basal Elkilab } & \text { G } & M, E\end{array}$


Table 1. Continued.

\begin{tabular}{|c|c|c|c|c|}
\hline Taxa & Species & Vernacular name & Life form & Uses \\
\hline \multicolumn{5}{|l|}{ Order Asparagales } \\
\hline \multicolumn{5}{|l|}{ Family Xanthorrhoeaceae } \\
\hline Subfamily Asphodeloideae & Aloe sinkatana Reynolds, J. S. & Sabar & G & M \\
\hline \multicolumn{5}{|l|}{ Family Amaryllidaceae } \\
\hline Subfamily Amaryllidoideae & Scadoxus multiflorus (Martyn) Raf. & Gash El Fil & G & $\mathrm{O}$ \\
\hline \multicolumn{5}{|l|}{ Family Asparagaceae } \\
\hline Subfamily Asparagoideae & Asparagus flagellaris (Kunth) Baker. & & $\mathrm{Ch}$ & $M, E$ \\
\hline \multicolumn{5}{|l|}{ Clade Monocots Commelinids } \\
\hline \multicolumn{5}{|l|}{ Order Arecales } \\
\hline \multicolumn{5}{|l|}{ Family Arecaceae } \\
\hline \multirow[t]{2}{*}{ Subfamily Coryphoideae } & Borassus aethiopum Mart. & Deleib & $\mathrm{Ph}$ & $M, E, T$ \\
\hline & Hyphaene thebaica (Linn.) Mart. & Dom & $\mathrm{Ph}$ & $M, E, T$ \\
\hline \multicolumn{5}{|l|}{ Order Commelinales } \\
\hline \multicolumn{5}{|l|}{ Family Commelinaceae } \\
\hline \multirow[t]{2}{*}{ Subfamily Commelinoideae } & Commelina benghalensis $\mathrm{L}$. & Bayad & Th & $\mathrm{Fd}$ \\
\hline & Commelina imberbis Ehrenb. ex Hassk. & Ibrig El Faki & Th & $\mathrm{Fd}$ \\
\hline \multicolumn{5}{|l|}{ Order Poales } \\
\hline \multirow[t]{4}{*}{ Cyperaceae } & Cyperus amabilis L & Seida & Th & M \\
\hline & Cyperus dives Delile. & Seida & G & M \\
\hline & Cyperus rotundus $\mathrm{L}$. & Seida & G & M \\
\hline & Kyllinga squamulata Vahl. & & Th & M \\
\hline \multicolumn{5}{|l|}{ Family Poaceae } \\
\hline \multirow[t]{2}{*}{ Subfamily Aristidoideae } & Aristida hordeacea Kunth. & Danab Elkadees & Th & $\mathrm{Fd}$ \\
\hline & Aristida mutabilis Trin. \& Rupr. & Milesa & Th & $\mathrm{Fd}$ \\
\hline Subfamily Bambusoideae & Oxytenanthera abyssinica (A. Rich.) Munro. & Ganna & $\mathrm{Ph}$ & $\mathrm{Fd}$ \\
\hline \multirow[t]{14}{*}{ Subfamily Chloridoideae } & Chloris pilosa Schumach. \& Thonn. & Abu Malhi & Th & $\mathrm{Fd}$ \\
\hline & Chloris virgata Swartz. & Um Faru & Th & $\mathrm{Fd}$ \\
\hline & Dactyloctenium aegyptium (L.) Willd. & Um Asabi & Th & $\mathrm{Fd}$ \\
\hline & Eragrostis aspera (Jacq.) Nees. & & Th & $\mathrm{Fd}$ \\
\hline & Eleusine indica (L.) Gaertn & & Th & $\mathrm{Fd}$ \\
\hline & Eragrostis cilianensis (All.) Vign. ex Janchen. & Gash Elnamil & Th & $\mathrm{Fd}$ \\
\hline & Eragrostis ciliaris (L.) R.Br. & & Th & $\mathrm{Fd}$ \\
\hline & Eragrostis japonica (Thunb.) Trin. & & Th & $\mathrm{Fd}$ \\
\hline & Eragrostis tremula Steud. & Bannu & Th & $\mathrm{Fd}$ \\
\hline & Microchloa indica (L.f.) P.Beauv. & & Th & $\mathrm{Fd}$ \\
\hline & Schoenefeldia gracilis Kunth. & Um farida & Th & $\mathrm{Fd}$ \\
\hline & Sporobolus festivus Hochst. ex A.Rich. & Korieb, Um Tuk & $\mathrm{He}$ & $\mathrm{Fd}$ \\
\hline & Tetrapogon cenchriformis (A. Rich.) W. D. Clyton. & Difra & Th & $\mathrm{Fd}$ \\
\hline & Tetrapogon tenellus (Roxb.) Chiov. & & Th & $\mathrm{Fd}$ \\
\hline \multirow[t]{14}{*}{ Subfamily Panicoideae } & Brachiaria xantholeuca (Hack.) Stapf. & Um Chir & Th & $\mathrm{Fd}$ \\
\hline & Cymbopogon nervatus (Hochst.) Chiov. & Nal & Th & $\mathrm{Fd}$ \\
\hline & Echinochloa rotundiflora Clayton. & Um-balil & Th & $\mathrm{Fd}$ \\
\hline & Hyparrhenia confinis var. nudiglumis (Hack.) Clayton & Um Sileila & Th & $\mathrm{Fd}$ \\
\hline & Ischaemum afrum (J.F. Gmel.) Dandy. & & Th & $\mathrm{Fd}$ \\
\hline & Melinis repens (Willd.) Zizka. & & Th & $\mathrm{Fd}$ \\
\hline & Panicum callosum Hochst. ex A.Rich. & & Th & $\mathrm{Fd}$ \\
\hline & Pennisetum nubicum (Hochst.) K.Schum. ex Engl & & Th & $\mathrm{Fd}$ \\
\hline & Pennisetum pedicellatum Tan. & Danab Elkadees, & Th & $\mathrm{Fd}$ \\
\hline & Pennisetum polystachion (L.) Schult. & & Th & $\mathrm{Fd}$ \\
\hline & Pennisetum purpureum Schumach. & Danab Elkalib & Th & $\mathrm{Fd}$ \\
\hline & Pennisetum ramosum (Hochst.) Schweinf. & & Th & $\mathrm{Fd}$ \\
\hline & Setaria pumila (Poir.) Roem. \& Schult. & Danab Elkalib & Th & $\mathrm{Fd}$ \\
\hline & Setaria verticillata (L.) P.Beauv & Abu elliseg & Th & $\mathrm{Fd}$ \\
\hline
\end{tabular}

\section{Clade Eudicots}

Order Ranunculales

Family Papaveraceae

Subfamily Papaveroideae

Argemone mexicana $\mathrm{L}$.

Ch $\quad M$

\section{Clade Core eudicots/Rosods}

\section{Order Vitales}

Family Vitaceae

Subfamily Vitoideae 
Table 1. Continued.

\begin{tabular}{|c|c|c|c|c|}
\hline Taxa & Species & Vernacular name & Life form & Uses \\
\hline \multicolumn{5}{|c|}{ Clade Core eudicots/Rosids/fabids (eurosids I) } \\
\hline \multicolumn{5}{|l|}{ Order Zygophyllales } \\
\hline \multicolumn{5}{|l|}{ Family Zygophyllaceae } \\
\hline \multirow[t]{2}{*}{ Subfamily Tribuloideae } & Balanites aegyptiaca (L.) Delile & Heglig & $\mathrm{Ph}$ & $\mathrm{T}, \mathrm{E}, \mathrm{Fd}, \mathrm{M}$ \\
\hline & Tribulus terrestris $\mathrm{L}$. & Direisa & Th & $\mathrm{Fd}$ \\
\hline \multicolumn{5}{|l|}{ Order Celastrales } \\
\hline \multicolumn{5}{|l|}{ Family Celastraceae } \\
\hline Subfamily Celastroideae & Maytenus senegalensis (Lam.) Exdl. & Yoi & $\mathrm{Ph}$ & M \\
\hline \multicolumn{5}{|l|}{ Order Fabales } \\
\hline \multicolumn{5}{|l|}{ Family Fabaceae } \\
\hline \multirow[t]{8}{*}{ Subfamily Caesalpinoideae } & Bauhinia rufescens Lam. & Kulkul & $\mathrm{Ph}$ & $\mathrm{Fu}, \mathrm{M}$ \\
\hline & Bauhinia reticulata DC. & Kharub & $\mathrm{Ph}$ & $\mathrm{E}, \mathrm{T}, \mathrm{Fd}$ \\
\hline & Cassia sieberana DC. & Um Kashu & $\mathrm{Ph}$ & $\mathrm{T}, \mathrm{Fu}, \mathrm{O}, \mathrm{M}$ \\
\hline & Chamaecrista mimosoides (L.) Greene & & $\mathrm{Ch}$ & M \\
\hline & Senna italica Mill. & Sin Elkalib & $\mathrm{Ch}$ & M \\
\hline & Senna obtusifolia (L.) Irwin \& Barneb & Kawal & $\mathrm{Ch}$ & $\mathrm{E}$ \\
\hline & Senna occidentalis (L.) Link. & Soreeb & $\mathrm{Ch}$ & $M, E$ \\
\hline & Tamarindus indica $\mathrm{L}$. & Aradeib & $\mathrm{Ph}$ & $\mathrm{E}, \mathrm{Fd}$ \\
\hline \multirow[t]{15}{*}{ Subfamily Mimosoideae } & Acacia gerrardii Benth. & Salgam & $\mathrm{Ph}$ & $\mathrm{Fu}, \mathrm{M}$ \\
\hline & Acacia mellifera (Vahl) Benth. & Kitir & $\mathrm{Ph}$ & $\mathrm{Fu},, \mathrm{Fd}$ \\
\hline & Acacia nilotica subsp. adstringens (Schum. \& Thonn.) Roberty. & Sunut & $\mathrm{Ph}$ & $\mathrm{T}, \mathrm{Fd}, \mathrm{M}$ \\
\hline & Acacia oerfota (Forssk.) Schweinf & Laot & $\mathrm{Ph}$ & M \\
\hline & Acacia polyacantha Willd. & Kakamut & $\mathrm{Ph}$ & $\mathrm{G}, \mathrm{Fd}, \mathrm{M}$ \\
\hline & Acacia senegal (L.) Willd. & Hashab & $\mathrm{Ph}$ & $\mathrm{G}, \mathrm{Fd}, \mathrm{Fu}$ \\
\hline & Acacia seyal Del. var. seyal & Talih & $\mathrm{Ph}$ & $\mathrm{G}, \mathrm{Fd}, \mathrm{Fu}, \mathrm{M}$ \\
\hline & Acacia sieberiana DC. & Kuk & $\mathrm{Ph}$ & $\mathrm{T}, \mathrm{Fu}$ \\
\hline & Acacia tortilis (Forssk.) Hayne subsp. raddiana (Savi) Brenan & Sayal & $\mathrm{Ph}$ & $\mathrm{Fd}$ \\
\hline & Albizia amara (Roxb.) Boiv. & Arad & $\mathrm{Ph}$ & $\mathrm{T}, \mathrm{Fu}$ \\
\hline & Albizia anthelmintica Brongn & Girfat Addud & $\mathrm{Ph}$ & $\mathrm{Fd}, \mathrm{M}$ \\
\hline & Albizia aylmeri Hutch. & Sireira & $\mathrm{Ph}$ & $\mathrm{T}$ \\
\hline & Dichrostachys cinerea (L.)White \& Arn. & Kadad & $\mathrm{Ph}$ & $\mathrm{Fu}, \mathrm{Fd}$ \\
\hline & Faidherbia albida (Del.) Chev. & Haraz & $\mathrm{Ph}$ & $\mathrm{T}, \mathrm{E}$ \\
\hline & Prosopis africana (Guill. \& Perr.) Taub. & Abu-surug & $\mathrm{Ph}$ & $\mathrm{Fd}$ \\
\hline \multirow[t]{20}{*}{ Subfamily Faboidae } & Alysicarpus glumaceus (Vahl) DC. & & $\mathrm{Ch}$ & $\mathrm{Fd}$ \\
\hline & Crotalaria lanceolata E. Mey. & & Th & $\mathrm{Fd}$ \\
\hline & Crotalaria goreensis Guill. \& Perr. & & $\mathrm{Ch}$ & $\mathrm{Fd}$ \\
\hline & Crotalaria ochroleuca G. Don & & $\mathrm{Ch}$ & $\mathrm{Fd}$ \\
\hline & Crotalaria ononoides Benth. & & $\mathrm{Ch}$ & $\mathrm{Fd}$ \\
\hline & Crotalaria senegalensis (Pers.) Bacle ex DC. & Sifeira & Th & $\mathrm{Fd}$ \\
\hline & Dalbergia melanoxylon Guill. \& Perr. & Babanous & $\mathrm{Ph}$ & $\mathrm{T}, \mathrm{M}, \mathrm{Fd}$ \\
\hline & Erythrina abyssinica DC. & Hab Elaroos & $\mathrm{Ph}$ & $\mathrm{O}, \mathrm{M}$ \\
\hline & Indigofera arrecta A. Rich. & & $\mathrm{Ch}$ & $\mathrm{Fd}$ \\
\hline & Indigofera hirsuta L. & & Th & $\mathrm{Fd}$ \\
\hline & Indigofera cordifolia B. Heyne ex Roth & & Th & $\mathrm{Fd}$ \\
\hline & Indigofera hochstetteri Baker & Sharaya & Th & $\mathrm{Fd}$ \\
\hline & Indigofera nummulariifolia (L.) Livera & & Th & $\mathrm{Fd}$ \\
\hline & Indigofera spicata Forssk. & & $\mathrm{Ch}$ & M \\
\hline & Lonchocarpus laxiflorus Guill. \& Perr. & Khashkhash Azrag & $\mathrm{Ph}$ & $\mathrm{T}, \mathrm{Fu}, \mathrm{M}$ \\
\hline & Mundulea sericea (Willd.) A. Chev. & Abu-galinga & $\mathrm{Ph}$ & M \\
\hline & Sesbania leptocarpa DC. & & $\mathrm{Ph}$ & $\mathrm{Fd}$ \\
\hline & Stylosanthes fruticosa (Retz.) Alston & & Th & $\mathrm{Fd}$ \\
\hline & Tephrosia uniflora Pers. & Irg El Tais, Abu Rassein & $\mathrm{Ch}$ & $\mathrm{Fd}$ \\
\hline & Zornia glochidiata Reichb. ex DC. & Sheleni & Th & $\mathrm{Fd}$ \\
\hline \multicolumn{5}{|l|}{ Order Rosales } \\
\hline \multirow[t]{2}{*}{ Family Rhamnaceae } & Ziziphus abyssinica Hochst. ex A. Rich. & Nabag Elfeel & $\mathrm{Ph}$ & $\mathrm{T}, \mathrm{E}, \mathrm{Fd}$ \\
\hline & Ziziphus spina-christi (L.) Desf. & Nabag, & $\mathrm{Ph}$ & $\mathrm{T}, \mathrm{E}, \mathrm{Fd}$ \\
\hline Family Ulmaceae & Celtis toka (Frossk.) Hepper \&Wood & Mohagria & $\mathrm{Ph}$ & $\mathrm{Fu}, \mathrm{M}$ \\
\hline \multirow[t]{4}{*}{ Family Moraceae } & Antiaris toxicaria subsp. africana (Engl.) C. C. Berg & & $\mathrm{Ph}$ & $\mathrm{T}$ \\
\hline & Ficus abutilifolia (Miq.) Miq. & Gumaiz & $\mathrm{Ph}$ & $\mathrm{E}$ \\
\hline & Ficus cordata subsp. salicifolia (Vahl) C.C. Berg Thumb. & Um Sisi & $\mathrm{Ph}$ & $M$ \\
\hline & Ficus glumosa Del. & Gumaiz & $\mathrm{Ph}$ & T \\
\hline
\end{tabular}


Table 1. Continued.

\begin{tabular}{|c|c|c|c|c|}
\hline Taxa & Species & Vernacular name & Life form & Uses \\
\hline & Ficus ingens Miq. & Gumaiz & $\mathrm{Ph}$ & $E$ \\
\hline & Ficus platyphylla Del. & Gumaiz & $\mathrm{Ph}$ & M \\
\hline & Ficus populifolia Vahl. & Gumaiz & $\mathrm{Ph}$ & $E$ \\
\hline & Ficus sycomorus $\mathrm{L}$. & Gumaiz & $\mathrm{Ph}$ & $\mathrm{E}$ \\
\hline & Ficus thonningii Blume. & Irkabi & $\mathrm{Ph}$ & M \\
\hline \multicolumn{5}{|l|}{ Order Cucurbitales } \\
\hline \multirow[t]{6}{*}{ Subfamily Cucurbitoideae } & Ctenolepis cerasiformis (Stocks) C. B. Clarke. & Sim Elter & Th & M \\
\hline & Cucumis metuliferus E.Mey. ex Naudin & Tibish Elkilab & Th & M \\
\hline & Cucumis prophetarum L. & Fagoos Elmarfaeen & Th & M \\
\hline & Lagenaria siceraria (Molina) Standley & Garaa or Bukhsa & Th & M \\
\hline & Luffa cylindrica (L.) M. J. Roem. & Leef & $\mathrm{Ch}$ & M \\
\hline & Momordica balsamina L. & Erra Iri & Th & M \\
\hline \multicolumn{5}{|l|}{ Order Malpighiales } \\
\hline \multirow[t]{4}{*}{ Subfamily Acalyphoideae } & Acalypha indica Linn. & & Th & M \\
\hline & Cephalocroton cordofanus Hochst. & & $\mathrm{Ch}$ & $\mathrm{Fd}$ \\
\hline & Dalechampia scandens L. var. cordofana (Webb) Müll. & Abu Liseig & Th & \\
\hline & Ricinus communis L. & Khirwa & $\mathrm{Ph}$ & M \\
\hline \multirow[t]{2}{*}{ Subfamily Crotonoideae } & Croton zambesicus Muell. & Um Ghilela & $\mathrm{Ph}$ & M \\
\hline & Jatropha aethiopica Muell. & Abu ofein & $\mathrm{Ch}$ & M \\
\hline \multirow[t]{5}{*}{ Subfamily Euphobioideae } & Euphorbia candelabrum Trémaux ex Kotschy & Shagar El-sim & $\mathrm{Ph}$ & M \\
\hline & Euphorbia heterophylla Wild. & & Th & M \\
\hline & Euphorbia hirta L. & Um libena & Th & M \\
\hline & Euphorbia prostrata Ait. Hort. & Um libena & Th & M \\
\hline & Euphorbia venenifica Tremaux ex Kotschy & Shagar El-sim & $\mathrm{Ph}$ & M \\
\hline Family Phyllanthaceae & Bridelia micrantha (Hochst) Baill. & Reel & $\mathrm{Ph}$ & $\mathrm{T}, \mathrm{Fu}, \mathrm{M}$ \\
\hline \multicolumn{5}{|l|}{ Family Passifloraceae } \\
\hline
\end{tabular}

Subfamily Passifloroideae

Adenia venenata Forssk

Kudur

\begin{tabular}{|c|c|c|}
\hline Sahab & $\mathrm{Ph}$ & $\mathrm{T}, \mathrm{Fu}, \mathrm{Fd}$, \\
\hline Shuheit & $\mathrm{Ph}$ & $\mathrm{Fu}, \mathrm{Fd}, \mathrm{N}$ \\
\hline Elhabeel & $\mathrm{Ph}$ & $\mathrm{T}, \mathrm{Fu}$ \\
\hline Habeel & $\mathrm{Ph}$ & Dyes \\
\hline Habeel & $\mathrm{Ph}$ & $M$ \\
\hline Habeel & $\mathrm{Ph}$ & $\mathrm{T}, \mathrm{Fu}, \mathrm{M}$ \\
\hline Ghibaish & $\mathrm{Ph}$ & $\mathrm{Fu}, \mathrm{M}$ \\
\hline \multirow[t]{2}{*}{ Subagh } & $\mathrm{Ph}$ & $\mathrm{T}, \mathrm{M}$ \\
\hline & $\mathrm{Ph}$ & $\mathrm{T}, \mathrm{M}$ \\
\hline Tamr Elfar & Th & M \\
\hline \multirow[t]{3}{*}{ Wad Am Elshai } & $\mathrm{Ph}$ & G \\
\hline & Th & $\mathrm{Fd}, \mathrm{E}$ \\
\hline & $\mathrm{Hy}$ & M \\
\hline Taragtarag & $\mathrm{Ph}$ & $\mathrm{G}, \mathrm{M}, \mathrm{Fu}$ \\
\hline Gaffal & $\mathrm{Ph}$ & $\mathrm{G}$, \\
\hline Leyun & $\mathrm{Ph}$ & $\mathrm{E}, \mathrm{M}$ \\
\hline Leyun & $\mathrm{Ph}$ & $\mathrm{T}$ \\
\hline Rutrut & $\mathrm{Ph}$ & $\mathrm{E}, \mathrm{M}, \mathrm{Fd}$ \\
\hline Elmilais, Amzaq & $\mathrm{Ph}$ & $\mathrm{Fu}, \mathrm{E}$ \\
\hline \multirow[t]{3}{*}{ El Homeid } & $\mathrm{Ph}$ & $T, E, M$ \\
\hline & $\mathrm{Ph}$ & $M$ \\
\hline & Th & M \\
\hline Fideila & $\mathrm{Ph}$ & $M$ \\
\hline Mahogani & $\mathrm{Ph}$ & $\mathrm{T}, \mathrm{M}$ \\
\hline Dimso & $\mathrm{Ph}$ & $\mathrm{T}$ \\
\hline
\end{tabular}

\section{Order Myrtales}

Family Combretaceae

Anogeissus leiocarpus (DC.) Guill. \& Perr.

Combretum aculeatum Vent.

Combretum collinum subsp. binderianum (Kotschy) Okafa.

Combretum glutinosum Perr. ex DC.

Combretum hartmannianum Schwein f. Beitr.

Combretum molle R.Br. ex G. Don.

Guiera senegalensis J. F. Gmel.

Terminalia brownii Fresen

Terminalia laxiflora Engl. \& Diels Monogr.

Family Lythraceae

Ammannia auriculata Willd.

Woodfordia uniflora (A.Rich.) Koehne.

Ludwigia erecta (L.) Hara.

Ludwigia adscendens (L.) Hara.

Boswellia papyrifera (Del.) Hochst.

Commiphora africana (A. Rich) Engl.

Lannea humilis (Oliv.) Engl.

Lannea fruticosa (Hochst. ex A. Rich.) Engl.

Lannea schweinfurthii (Engl.) Engl.

Lannea schimperi (Hochst. ex. A. Rich.) Engl.

Sclerocarya birrea (A. Rich.) Hochst.

Family Sapindaceae

Allophylus africanus P. Beauv.

Cardiospermum halicacabum L.

Family Rutaceae

Vepris nobilis (Del.) Mziray

Family Meliaceae

Khaya senegalensis (Desr.) A. Juss.

Trichilia emetica Vahl.

Order Malvales

Family Malvaceae

Subfamily Bombacoideae

Adansonia digitata Linn.

Tabaldi

$\mathrm{Ph}$ 
Table 1. Continued.

\begin{tabular}{|c|c|c|c|c|}
\hline Taxa & Species & Vernacular name & Life form & Uses \\
\hline \multirow[t]{8}{*}{ Subfamily Grewiodeae } & Corchorus pseudocapsularis Schweinf. & & Th & $\mathrm{Fd}$ \\
\hline & Corchorus tridens $\mathrm{L}$. & Khodra & $\mathrm{Ch}$ & $\mathrm{E}$ \\
\hline & Grewia bicolor Juss. & Basham & $\mathrm{Ph}$ & $\mathrm{Fu}, \mathrm{T}, \mathrm{Fd}$ \\
\hline & Grewia flavescens Juss. & Khlekhsan & $\mathrm{Ph}$ & $\mathrm{Fu}, \mathrm{E}, \mathrm{Fd}, \mathrm{M}$ \\
\hline & Grewia mollis Juss. & Basham & $\mathrm{Ph}$ & $\mathrm{Fu}, \mathrm{E}$ \\
\hline & Grewia tenax (Forsk.) Fiori. & Elgudiem & $\mathrm{Ph}$ & $\mathrm{Fu}, \mathrm{E}, \mathrm{Fd}$ \\
\hline & Grewia villosa Willd. & Tikko & $\mathrm{Ph}$ & $\mathrm{Fu}, \mathrm{E}, \mathrm{M}$ \\
\hline & Triumfetta pentandra J.M. Garg & Abu liseig & $\mathrm{Ch}$ & $\mathrm{Fd}, \mathrm{E}, \mathrm{M}, \mathrm{Fd}$, \\
\hline \multirow[t]{9}{*}{ Subfamily Malvoideae } & Abelmoschus esculentus (L.) Moench & Weika & Th & $E$ \\
\hline & Abutilon angulatum (Guill. \& Perr.) Mast. & Amboru & $\mathrm{Ch}$ & $\mathrm{Fb}$ \\
\hline & Azanza garckeana (F. Hoff.) Exell \& Hillcoat. & Nakhgar & $\mathrm{Ph}$ & $\mathrm{E}, \mathrm{G}$ \\
\hline & Hibiscus diversifolius Jacq. & & $\mathrm{Ch}$ & $\mathrm{Fb}, \mathrm{M}$ \\
\hline & Hibiscus vitifolius L. & & Th & $\mathrm{Fb}, \mathrm{M}$ \\
\hline & Hibiscus cannabinus L. & Kirkaiya, Kenaf, Til & $\mathrm{Ch}$ & $\mathrm{Fb}$ \\
\hline & Sida alba Linn. & Um-shadia & $\mathrm{Ch}$ & M \\
\hline & Sida cordifolia L. & Nayada & $\mathrm{Ch}$ & M \\
\hline & Wissadula amplissima (L.) R. E. Fr. Kongl. & & $\mathrm{Ch}$ & M \\
\hline \multirow[t]{2}{*}{ Subfamily Sterculiodeae } & Sterculia setigera Del. & Tartar & $\mathrm{Ph}$ & $\mathrm{G}, \mathrm{M}$ \\
\hline & Waltheria indica $\mathrm{L}$. & Erg Elnal & $\mathrm{Ch}$ & M \\
\hline \multicolumn{5}{|l|}{ Order Brassicales } \\
\hline Family Salvadoraceae & Dobera glabra (Forssk.) Juss. ex Poir. & Mikah & $\mathrm{Ph}$ & $\mathrm{Fd}$ \\
\hline \multirow[t]{7}{*}{ Family Capparaceae } & Boscia angustifolia A. Rich. & Elsereh, Serha & $\mathrm{Ph}$ & $\mathrm{Fu}, \mathrm{E}, \mathrm{Fd}, \mathrm{M}$ \\
\hline & Boscia senegalensis Lam. & Mikhiet, Kursn & $\mathrm{Ph}$ & $\mathrm{E}, \mathrm{Fd}, \mathrm{M}$ \\
\hline & Cadaba rotundifolia Forssk. & Kurmut & $\mathrm{Ph}$ & $\mathrm{Fd}, \mathrm{M}$ \\
\hline & Capparis deciduas (Forsk.) Edgew. & Tundub & $\mathrm{Ph}$ & $\mathrm{Fu}, \mathrm{E}, \mathrm{Fd}$ \\
\hline & Capparis tomentosa Lam. & Murdu & $\mathrm{Ph}$ & $\mathrm{Fd}, \mathrm{M}$ \\
\hline & Crateva adansonii DC. & Dabkar & $\mathrm{Ph}$ & $\mathrm{T}, \mathrm{Fu}, \mathrm{Fd}$ \\
\hline & Maerua angolensis DC. & Shagar Eldoud & $\mathrm{Ph}$ & $\mathrm{T}, \mathrm{Fd}, \mathrm{M}$ \\
\hline Family Cleomaceae & Cleome gynandra $\mathrm{L}$. & Tamaleka & $\mathrm{Ch}$ & $E$ \\
\hline
\end{tabular}

\section{Clade Eudicots}

\section{Order Santalales}

Family Olacaceae

Family Loranthaceae

Order Caryophyllales

Family Tamaricaceae

Family Plumbaginaceae

Subfamily Plumbaginoideae

Family Caryophyllaceae

Family Amaranthaceae

Subfamily Amaranthoideae

Ximenia americana $\mathrm{L}$.

Tapinanthus globiferus (A. Rich.) Tiegh.

Kalto

Anaba

$\mathrm{Ph}$

$\mathrm{E}, \mathrm{Fd}, \mathrm{M}$

Tamarix nilotica (Ehrenb.) Bunge

Tarfa

$\mathrm{Fd}, \mathrm{M}$

Plumbago zeylanica L.

Polycarpaea corymbosa (L.) Lam.

Um Fara

Achyranthes aspera L.

Amaranthus graecizans L.

Amaranthus hybridus L.

Celosia argentea $\mathrm{L}$.

Celosia trigyna $\mathrm{L}$.

Pupalia lappacea (Linn) Juss.

Subfamily Gomphrenoideae

Alternanthera pungens Kunth.

Zaleya pentandra (L.) C.

Subfamily Sesuvioideae

Boerhavia africana Lour.

Boerhavia chinensis (L.) Rottb.

Boerhavia diffusa $\mathrm{L}$.

Boerhavia erecta L.

Family Molluginaceae

Glinus lotoides L.

Family Portulacaceae

Portulaca oleracea $\mathrm{L}$.

$\begin{array}{lll}\text { Fakhah } & \text { Th } & \text { M } \\ \text { Lisan Elteir } & \text { Th } & \text { Fd } \\ \text { Khadiga kouro } & \text { Th } & \text { Fd } \\ \text { Danab Elkalib } & \text { Ch } & \text { O, M } \\ & \text { Th } & \text { M } \\ \text { Abu Luseg } & \text { Ch } & \text { M } \\ \text { Haskaneet } & \text { Ch } & \text { Fd } \\ \text { Rigl Eltir } & & \\ \text { Abu Libin } & \text { Ch } & \text { M, Fd } \\ & \text { Ch } & \text { M } \\ \text { Um Shiraya } & \text { Ch } & \text { E, M } \\ \text { Gashayt Elter } & \text { Th } & \text { M } \\ \text { Raba'a } & \text { Th } & \text { E, Fd, M } \\ \text { Rigla } & \text { Th } & \text { M, Fd } \\ & \text { Th } & \text { E }\end{array}$

\section{Clade Eudicots/Asterids}

Order Ericales

Family Ebenaceae

Diospyros mespiliformis Hochst. ex A. DC

Goghan

$\mathrm{Ph}$

$\mathrm{T}, \mathrm{E}, \mathrm{Fd}, \mathrm{M}$

\section{Clade Eudicots/Asterids/Lamids (euasterids I)}


Table 1. Continued.

\begin{tabular}{|c|c|c|c|c|}
\hline Taxa & Species & Vernacular name & Life form & Uses \\
\hline \multirow[t]{5}{*}{ Subfamily Ixoroideae } & Catunaregam nilotica (Stapf.) Tirveng. & Shagart Elmarfaein & $\mathrm{Ph}$ & $\mathrm{Fu}, \mathrm{M}$ \\
\hline & Feretia apodanthera Del. & Shai Elbitera & $\mathrm{Ph}$ & $\mathrm{E}$, \\
\hline & Gardenia ternifolia var. jovis-tonantis (Welw.) Verdc. & Baggis & $\mathrm{Ph}$ & M \\
\hline & Meyna tetraphylla (Schweinf. ex Hiern) Robyns. & Simeim & $\mathrm{Ph}$ & $\mathrm{E}$ \\
\hline & Vangueria madagascariensis Gmel. & Kirkir & $\mathrm{Ph}$ & $E$ \\
\hline Subfamily Rubioideae & Oldenlandia corymbosa L. & Bushari & Th & M \\
\hline Family Loganiaceae & Strychnos innocua Del. & Um Bikhesa & $\mathrm{Ph}$ & $\mathrm{E}, \mathrm{T}, \mathrm{Fd}, \mathrm{M}$ \\
\hline \multicolumn{5}{|l|}{ Family Apocynaceae } \\
\hline Subfamily Apocynoideae & Adenium obesum (Forssk.) Roem \& Schult. & Shagar Elsim & $\mathrm{Ph}$ & $\mathrm{O}, \mathrm{M}$ \\
\hline \multirow[t]{2}{*}{ Subfamily Asclepiadoideae } & Calotropis procera (Aiton) Dryand. & Elushar & $\mathrm{Ph}$ & M \\
\hline & Pergularia daemia (Forssk.) Cheov. & & $\mathrm{Ch}$ & \\
\hline \multicolumn{5}{|l|}{ Unplaced in an order } \\
\hline Family Boraginaceae & Cordia africana Lam. & Gimbeel & $\mathrm{Ph}$ & $\mathrm{T}, \mathrm{E}$ \\
\hline \multirow[t]{6}{*}{ Family Convolvulaceae } & Astripomoea lachnosperma (Choisy) A. Meeuse & Um Ghaleila & $\mathrm{Ch}$ & M \\
\hline & Ipomoea cordofana Choisy. & Tabar & Th & $\mathrm{Fd}$ \\
\hline & Ipomoea sinensis (Desr.) Choisy. & Hantut & Th & $\mathrm{Fd}$ \\
\hline & Ipomoea eriocarpa $\mathrm{R} . \mathrm{Br}$. & & Th & $\mathrm{Fd}$ \\
\hline & Ipomoea calophylla Fenzl. & Um She`era & Th & M \\
\hline & Merremia aeygptia (L.) Urb. & Erg M`oterash & Th & M \\
\hline \multicolumn{5}{|l|}{ Family Solanaceae } \\
\hline \multirow[t]{6}{*}{ Subfamily Solanoideae } & Datura innoxia Mill. & Sikeran & $\mathrm{Ch}$ & M \\
\hline & Physalis peruviana $\mathrm{L}$. & kurm kurum & $\mathrm{Ch}$ & M \\
\hline & Solanum coagulans Forssk. & Gubein & $\mathrm{Ch}$ & M \\
\hline & Solanum hastifolium Hochst. ex Dunal. & & $\mathrm{Ch}$ & M \\
\hline & Solanum incanum L. & Gubein & $\mathrm{Ch}$ & M \\
\hline & Solanum nigrum $\mathrm{L}$. & Um Gibin gibin & Th & M \\
\hline Family Scrophulariaceae & Striga hermonthica (Del.) Benth. & Bouda & Th & M \\
\hline \multirow[t]{2}{*}{ Family Pedaliaceae } & Rogeria adenophylla J.Gray ex Del. & & $\mathrm{Ch}$ & M \\
\hline & Sesamum radiatum Schumach. & Simsim Eljebal & Th & $E, M$ \\
\hline \multirow[t]{3}{*}{ Family Lamiaceae } & Leonotis nepetifolia (L.) R. Br. & Kashaw & $\mathrm{Ch}$ & M \\
\hline & Leucas martinicensis R. Br. & Baraw & Th & M \\
\hline & Ocimum americanum $\mathrm{L}$. & Rehan & $\mathrm{Ch}$ & M \\
\hline \multicolumn{5}{|l|}{ Family Acanthaceae } \\
\hline \multirow[t]{8}{*}{ Subfamily Acanthoideae } & Barleria prionitis $\mathrm{L}$ & & $\mathrm{Ch}$ & M \\
\hline & Barleria steudneri C. B. & & $\mathrm{Ch}$ & \\
\hline & Blepharis linariifolia Pers. & Baghel & Th & $\mathrm{Fd}$ \\
\hline & Dicliptera verticillata (Forssk.) Christens. & & Th & M \\
\hline & Hypoestes verticillaris (L. f.) Solander ex Roem. \& Schult. & & $\mathrm{Ch}$ & $\mathrm{E}, \mathrm{M}, \mathrm{Fd}$ \\
\hline & Hygrophila auriculata (Schumach.) Heine. & Abu Shweika & $\mathrm{Ch}$ & M \\
\hline & Monechma debile (Forssk.) Nees. & & Th & \\
\hline & Peristrophe paniculata (Forssk.) Brummitt. & Abu Rukab & Th & $\mathrm{M}, \mathrm{Fd}$ \\
\hline \multirow[t]{2}{*}{ Family Bignoniaceae } & Kigelia africana (Lam.) Banth. & Um Shutour & $\mathrm{Ph}$ & $\mathrm{T}, \mathrm{M}$ \\
\hline & Stereospermum kunthianum Cham. & Khashkhash abiad & $\mathrm{Ph}$ & $\mathrm{Fu}, \mathrm{M}, \mathrm{Fd}$ \\
\hline \multirow[t]{2}{*}{ Family Verbenaceae } & Clerodendrum capitatum (Willd.) Schumach. \& Thonn. & & $\mathrm{Ch}$ & M \\
\hline & Vitex doniana Sweet. & Um-Togulgul & $\mathrm{Ph}$ & $\mathrm{T}, \mathrm{E}, \mathrm{M}$ \\
\hline
\end{tabular}

Clade Core Eudicots/ Asterids/Campanulids (euasterids II)

Order Asterales

Family Asteraceae

Subfamily Asteroideae

Acanthospermum hispidum DC

Blainvillea gayana Cass.

Bidens bipinnata $\mathrm{L}$.

Bidens chippii (M.B.Moss) Mesfin.

Chrysanthellum indicum DC.

Dicoma tomentosa Cass.

Goniocaulon indicum C. B. Clarke

Pentanema indicum (L.) Y. Ling

Pseudoconyza viscosa (Mill.) D’Arcy

$\begin{array}{lll}\text { Hurab hawsa } & \text { Th } & \text { M } \\ & \text { Th } & \text { M } \\ \text { Th } & \text { E, M } \\ \text { Th } & \\ \text { Um shoka } & \text { Th } & \text { M } \\ & \text { Ch } & \text { M } \\ & \text { Ch } & \text { M } \\ & \text { Th } & \text { M } \\ & & \text { E, M }\end{array}$


Table 1. Continued.

\begin{tabular}{|c|c|c|c|c|}
\hline Taxa & Species & Vernacular name & Life form & Uses \\
\hline & Xanthium strumarium subsp. brasilicum (Vell.) O. Bolos \& Vigo & Ramtook & $\mathrm{Ch}$ & M \\
\hline & Sonchus oleraceus (L.) L. & Moleita & Th & $M$ \\
\hline & Vernonia adoensis Schultz-Bip. ex Walp. & & $\mathrm{Ch}$ & $\mathrm{M}, \mathrm{O}$ \\
\hline & Vernonia amygdalina Del. & & $\mathrm{Ph}$ & M \\
\hline \multicolumn{5}{|l|}{ Order Apiales } \\
\hline \multicolumn{5}{|l|}{ Family Apiaceae } \\
\hline Subfamily Saniculoideae & Steganotaenia araliacea Hochst. & Damin Ashara & $\mathrm{Ph}$ & $\mathrm{Fu}, \mathrm{M}$ \\
\hline
\end{tabular}

